\title{
Observation Statements
}

\author{
Dr. Thomas W. Smythe \\ Retired Associate Professor of Philosophy \\ North Carolina Central University \\ North Carolina \\ thomaswsmythe@yahoo.com
}

\begin{abstract}
It is generally agreed that science must be based on observations or sense perception. For a theory or hypothesis to be scientific it must be empirically verifiable or falsifiable via the five sense modalities. This paper reprises an article by Fred Dretske that provides conditions under which we have an observation term. It goes on to present and defend an Alternative Approach comparing and contrasting the alternative to Dretske's account.
\end{abstract}

KeyWords: Empirical; Hypothesis; Observation

\section{INTRODUCTION}

It is a fundamental part of our concept of the scientific enterprise that it aims at building up a certain kind of knowledge that has an empirical basis. 'Empirical' means 'knowledge gained through the five sense modalities. When we say science has an empirical basis we at least mean that any hypothesis $(\mathrm{H})$ must somehow be confronted with empirical data, and that the $\mathrm{H}$ stands or falls by being faced with the data. If this is to make sense, we must know what is and what is not an empirical datum.

In philosophy of science the problem is put in terms of the language of science, in terms of identifying an observation statement (O-statement) or report. This is because in scientific practice we have to process the data in linguistic terms before we can use the data in any way as evidence. We need to formulate results of observations and see what logical bearing they have on Hs. Here are two problems in the epistemology of science.

1. How do O-statements relate to Hs so that their truth or falsity reflects on the acceptability of $\mathrm{H}$ ?

2. What is it (the conditions) for a statement to be related to sense experience? In virtue of what is a statement an 0-statement?

\section{DiscUSSION}

This problem has practical importance, especially in the social sciences. There are many disputes that go on in social sciences over what is acceptable as data. In psychology the introspection-behaviorist controversy is famous. One school or theoretical orientation may accept a statement as an observations report while another says it is an interpretation. Consider psychoanalytic theory. Some say the analyst A observed the patient P become hostile; others say the P said, "You never believe anything I say," etc., and the A interpreted this as hostility. Another example in psychology is rat learning experiments. E. C. Tolman and Edwin R. Guthrie disagree over how we should conceptualize behavior as a dependent variable. Guthrie construed behavior in terms of bodily movements. "The rat turned around" would count as an 0 -statement. Tolman said we can report what the rat is doing in purposive terms. Consider the following two statements.

"The rat is exploring the maze."

"The rat was running towards the box."

Guthrie might call these interpretations. 
In getting at what counts as an O-statement we will aim at statements that are independently warranted. An independently warranted statement is one that can be accepted without depending on anything else. This is because we want to get out of the chain of statements whose warrant depends on other statements. We want statements that we can begin with as the building blocks of science. What makes us accept 0 -statements? Why are some statements $\mathrm{O}$-statements and not others? There is a tradition in the philosophy of science which tries to isolate $\mathrm{O}$-statements in terms of some honorific epistemological features. Philosophers since Rene Descartes have attempted to reconstruct and isolate some foundation that gives us certainty. In recent philosophy of science the tradition has been reserved by trying to isolate Simon pure 0-statements that are basic. This story has never been worked out to any satisfactory way. People cannot show how we can get from the contents of our conscious fields to physical things talked about in the sciences. Let us dub this tradition the Absolute Foundation View. We shall abandon this quest in favor of a more contextualist epistemology of science. We will accept statements made on the basis of sense perception as true unless there is some good reason for doubting them. We treat any such statements as defensible and subject to being given up.

Let us now turn to an important paper by Fred Dretske that attempts to mark off observation terms that can serve as the basis for empirical science. (Dretske, 1964) Dretske begins with the notion of an observation term. According to the author, we can get at a class of observation terms by examining the conditions under which "S sees that $\mathrm{x}^{\prime \prime}$ is true.

$S$ sees that $x$ if and only if

1. $\mathrm{x}$ exists

2. $\mathrm{S}$ is looking at $\mathrm{x}$

3. $\mathrm{S}$ is thoroughly familiar with $\mathrm{x}$ and its relations to other phenomena in some theory $\mathrm{T}$

4. Within theory $\mathrm{Y}$ it is a routine assumption that $\mathrm{X}$

Now let us look at these conditions more closely.

1. $x$ exists

This is a success sense of the verb "to see." If $\mathrm{S}$ looks for $\mathrm{x}$, and there is no $\mathrm{x}$, we would not say that he saw $\mathrm{x}$, or sees that $\mathrm{x}$.

\section{2. $\mathrm{S}$ is looking at $\mathrm{x}$}

"I see that today is Tuesday" or "I see the stock market is falling" are not perceptual uses of 'see,' because there is nothing we can look at, or have affect our sensory receptors as a result of turning in the right direction. 1 and 2 are truth conditions because if they are not true of S, we can say $S$ does not see that $x .3$ and 4 are routine assumptions within a theory. This accounts for why meteorologists can see that a cold front is approaching while the layman sees that there are clouds approaching; or how a scientist can see that a red object has a certain wave length.

It seems to us that Dretske fails to distinguish between perceptual objects and perceptual facts. Consider the following perceptual idioms.

1. "I see an x-ray tube."

2. "I see that an x-ray tube is on the table."

In 1. 'seeing' takes a substantive as object. In 2. 'seeing' takes a propositional clause as the object. In 1. we have a locution best suited for talking about perceiving objects; in 2. it is best suited for perceiving facts. This distinction is important for science. In 1 . we are ready to say that subject $S$ saw object $x$ even if he cannot recognize it, 
or hasn't got the foggiest idea what it is. "Seeing that" locutions are more sensitive to perceptual variations. For purposes of specifying the empirical basis of science, we need to restrict ourselves to the specification of perceptual facts the perceiver himself would use. It is facts and observation of facts that are more important for a discussion of empirical data. The kind of observation that is important to philosophy of science is that which can be formulated in $\mathrm{O}$-statements, and these are facts. $\mathrm{O}$-statements are perceptions that

"Seeing x" differs from O-statements in two ways. First, S can be said to see x even though he doesn't know what it is or have the relevant concept. For example, a native sees an automobile. Second, if we agree that $\mathrm{x}$ 's are observable, that doesn't tell us which facts about $\mathrm{x}$ can be asserted on the basis of direct observation. Put linguistically, this gives us subjects, but not predicates. But the notion of an 0-predicate or statement is important for philosophy of science.

It is now time to give a Different Approach to the notion of an O-statement. "X is P" is an O-statement if and only if " $\mathrm{X}$ is $\mathrm{P}$ " is warranted if asserted by some subject $\mathrm{S}$, and if

1. $S$ is in sensory range of $x$

2. S's statement was prompted by sensory stimulation from $\mathrm{x}$

3. S has the concepts of $\mathrm{X}$ and $\mathrm{P}$

4. $\mathrm{S}$ is in normal conditions

5. S's environment is conducive to accurate observation

This analysis specifies an 0 -statement so that we do not require it to be true to be an 0 -statement. This contrasts with the success of 'see.' These five truth conditions in the Alternative Approach are s-conditions (Dretske's term) that are background assumptions that are relatively uncontroversial in ordinary perceptual claims. When more or more of these conditions fail to hold we will withdraw the view that "X is P" is an O-statement.

We are rejecting traditional elements of s-conditions, such as indubitability and not being inferred from anything else. But as a matter of fact $\mathrm{O}$-statements will be relatively incorrigible (Dretske: routine assumption) and basic. They are basic in the sense that " $\mathrm{X}$ is $\mathrm{P}$ " is warranted in itself, independently of anything else.

It is important to honor the distinction between what is and what is not observable. In the scientific enterprise we want to be able to separate out aspects of knowledge that are over and above knowledge that is employed by virtue of sense experience. We are distinguishing between different kinds of knowledge, that which is implied by observation and that which goes beyond this.

In condition 3 we differ from the author in that we don't say that " $\mathrm{X}$ is $\mathrm{P}$ " is an 0 -statement unless the only knowledge involved is that which is involved in the concept we are applying. In any given context Dretske allows in other knowledge. As an illustration of this, take the statement that "A cold front is approaching." Where the author says it is an 0-statement, we say one has to have the concept so he can recognize it by sight. Otherwise, it is not an $\mathrm{O}$-statement. One must also know what the visual signs of a cold front are, in addition to the five conditions. The difference is important and needs incorporation into drawing the distinction. The Alternative Approach has the distinction of highlighting the above distinction. It should be said that on both accounts there is no a priori limit on what can and cannot be observed. But Dretske lets in more statements in any given context. Our standards are such that we have different treatments for various predicates, that is, we do or do not take the observer's say so. The way we sort predicates out in this fashion is not independent of the other things we know and believe. This is the result of abandoning the Absolute Foundations View with the notion of absolute perceptual certainties.

Volume 2

Page 3 
We can justify distinguishing between observation and non-observation reports the way we do. It is a sociological fact that in our epistemic standards in science we have different treatments for various predicates. Consider the following statements.

1. S said, "My father is trying to destroy my self-confidence."

2. $\mathrm{S}$ is projecting hostility onto his father.

In 1 . we have the evidence of an utterance (0-statement), but in 2. we demand more; we demand some theory and the ability to detect such things. We justify this difference by pointing out that when "X is $\mathrm{P}$ " statements are made under s-conditions, the statements usually turn out to be correct. If we adopt the policy of trusting observers, we aren't going to be wrong very often. If we adopt the same policy for 2 . we have no such assurance for being right.

Finally, we have not treated self-reports. This is because introspection or our inner self-knowledge is too large a topic to be treated in this short space.

\section{REFERENCE}

1. Fred Dretske, (1964), “Observation Terms," Philosophical Review, Vol. 75.

Citation: Dr. Thomas W. Smythe, "Observation Statements". American Research Journal of History and Culture; Volume 2, 2016; pp:1-4

Copyright (c) 2016 Dr. Thomas W. Smythe, This is an open access article distributed under the Creative Commons Attribution License, which permits unrestricted use, distribution, and reproduction in any medium, provided the original work is properly cited. 